九州大学学術情報リポジトリ

Kyushu University Institutional Repository

Design of a Semi-confocal Fluorescence Microscope for Observing Excitation Spectrum of Soluble Molecules Adsorbed at the Air/water Interface

Yang, Haiya

Interdisciplinary Graduate School of Engineering Sciences, Kyushu University

Harata, Akira

Faculty of Interdisciplinary Graduate School of Engineering Sciences, Kyushu University : Professor

https://doi.org/10.5109/1544074

出版情報: Evergreen. 2 (2)，pp. 1-4，2015-09. Green Asia Education Center バージョン:

権利関係 : 


\title{
Design of a Semi-confocal Fluorescence Microscope for Observing Excitation Spectrum of Soluble Molecules Adsorbed at the Air/water Interface
}

\author{
Haiya Yang ${ }^{1}$, Akira Harata ${ }^{2, *}$ \\ ${ }^{1}$ Interdisciplinary Graduate School of Engineering Sciences, Kyushu University, Japan \\ ${ }^{2}$ Faculty of Interdisciplinary Graduate School of Engineering Sciences, Kyushu University, Japan
}

*Author to whom correspondence should be addressed,

E-mail: harata@mm.kyushu-u.ac.jp

(Received June25, 2015; accepted July 13, 2015).

\begin{abstract}
A semi-confocal fluorescence microscope is designed and constructed aiming to observe fluorescence excitation spectrum of soluble molecules adsorbed at the air/water interface. With a conventional confocal fluorescence microscope, the target molecules of pyrene arises some difficulties: excitation wavelength in deep ultraviolet forces low transmission performance of microscopic objectives and low wavelength-tunable performance of an excitation laser. A total internal reflection illumination with a light-guide coupled monochromated-xenon lamp is combined with the confocal fluorescence microscope. Theoretical feasibility and performance of the microscope are discussed with preliminary results obtained.
\end{abstract}

Keywords: ScFM, the air/water interface, excitation spectrum, fluorescence spectrum, pyrene.

\section{Introduction}

It is greatly important to understand the air/water interface in physical, analytical, and environmental chemistry. ${ }^{1)}$ One of topics is to study physical and chemical properties of molecules such as surfactants and water-soluble solutes adsorbed at the air/water interface. ${ }^{2)}$ Although computer-based calculation gives a large progress for molecular view of the interface recently, some difficulties make experimental approaches still uneasy, especially in observing solute molecules adsorbed at the air/water interface highly sensitively and interfaceselectively. Confocal fluorescence microscope (CFM) has been applied to observe fluorescence emission of watersoluble rhodamine dyes adsorbed at the air/water interface even under the surface density of solute molecules being $1-10^{3}$ molecule $/ \mu \mathrm{m}^{2}{ }^{3-5)}$ However, it is still difficult to surface-selectively observe absorption spectra of watersoluble solute molecules adsorbed at the air/water interface, especially for aromatic hydrocarbons having no visible light-absorption. Combining with its corresponding fluorescence emission spectra, absorption spectra of molecules reveal physical and chemical properties of the air/water interface, when the air/water interface is considered as a common organic solvent. $\left.{ }^{6}\right)$ Even if it is hard to observe absorption spectra directly for the solute molecules adsorbed at the air/water interface mainly because of lacks in detection sensitivity, fluorescence excitation spectra could be measured in behalf of absorption spectra.
Poly-aromatic cyclic hydrocarbons (PAHs) are ubiquitous in our atmosphere, toxic to our environment, and absorb ultraviolet light. ${ }^{7)}$ Pyrene of the target molecule in this research is one of the typical PAHs and has no visible light-absorption as is the most of environmentally important PAHs with a small molecular weight. When a conventional CFM is applied to observe the fluorescence excitation spectra of the target, there arise some difficulties: excitation wavelength in deep ultraviolet forces to use a microscopic objectives with low transmission performance and a pulsed excitation laser with low wavelength-tunable performance in ultraviolet. These difficulties are so common in observing environmentally toxic molecules because these molecules generally have little visible light-absorption. Therefore, a new type of fluorescence microscope is counted on observing excitation spectra of PAHs molecules adsorbed at the air/water interface.

In this work, a semi-confocal fluorescence microscope ( $\mathrm{ScFM})$ is designed and constructed for aiming to observe fluorescence excitation spectra of soluble molecules adsorbed at the air/water interface. A total internal reflection illumination with a light-guide coupled monochromated-xenon lamp is combined with the confocal fluorescence microscope. Performance of the ScFM is evaluated and discussed. The emission characteristics of the excitation light is examined. A preliminary result about emission spectrum of pyrene molecules adsorbed at the air/water interface is obtained with this microscope. 


\section{Experimental}

\subsection{Apparatus}

The experimental setup of ScFM is schematically illustrated in Fig.1, which is similar to that of CFM in our past work, ${ }^{3)}$ except for an additional incoherent ultraviolet excitation beam. Adjusting optical paths in ScFM is also completed by referring to that in CFM. A beam of polychromatic ultraviolet light emitted from a xenon lamp (Max-303UV, Asahi Spectra Co.,Ltd.) is dispersed by a monochromator (CT-10, Jasco Corp.) installing a grating with a 600 grooves $/ \mathrm{mm}$ ruling and a blazing wavelength of $300 \mathrm{~nm}$, into monochromatic ultraviolet light with a tunable wavelength from $260 \mathrm{~nm}$ to $400 \mathrm{~nm}$. This monochromator is connected with a light guide (Quartz Light Guide, Asahi Spectra Co.,Ltd.) by means of a light guide connecter (CMS100-F.S $\varnothing 20$, Asahi Spectra Co.,Ltd.). The light guide transmits this tunable monochromatic ultraviolet light from the monochromator into a collimator lens (RLQL80-1, Asahi Spectra Co.,Ltd.), which clamped by an extension clamp in an iron support. The tunable monochromatic ultraviolet light emitted from the collimator lens illuminates at the interface of $1 \times 10^{-8} \mathrm{M}$ pyrene aqueous solution (thickness, $2 \mathrm{~mm}$ ) in a total internal reflection geometry. Pyrene solution is loaded in a quartz cell $(40 \mathrm{~mm} \times 40 \mathrm{~mm} \times 20 \mathrm{~mm})$. Fluorescence emissions generated at the air/water interface are collected with fluorescence collection system made up of an objective lens (UPLFLN40C, Olympus), a lens (SLSQ20-50p, Sigma Koki) and a pinhole (diameter, $200 \mu \mathrm{m}$ ). The only focus point of this microscope is existing in this fluorescence collection system, different from CFM that also has another focus point existing in illuminating the sample with laser. So we call it a semi-confocal fluorescence microscope. A polychromatic spectroscope (PMA-100, Hamamatsu; 300 grooves $/ \mathrm{mm}, 600 \mathrm{~nm}$ ) receives fluorescence emissions and complete photoelectric conversion with an image intensifier unit and a charge coupled device inside, under experimental conditions of exit slit of $10 \mu \mathrm{m}$, entrance slit of $100 \mu \mathrm{m}$, and accumulation time of $20 \mathrm{~s}$. An electricity-based cooling system is induced to keep temperature of the charge coupled device at $-14.5{ }^{\circ} \mathrm{C}$ to reduce noise. A personal computer records and analyzes fluorescence signals with its software (U8167-02, Ver 1.3.0). Although fluorescence emissions generated in bulk are collected simultaneously, their intensity are reasonable to be reduced substantially, and thus a surface-selective collection is achieved.

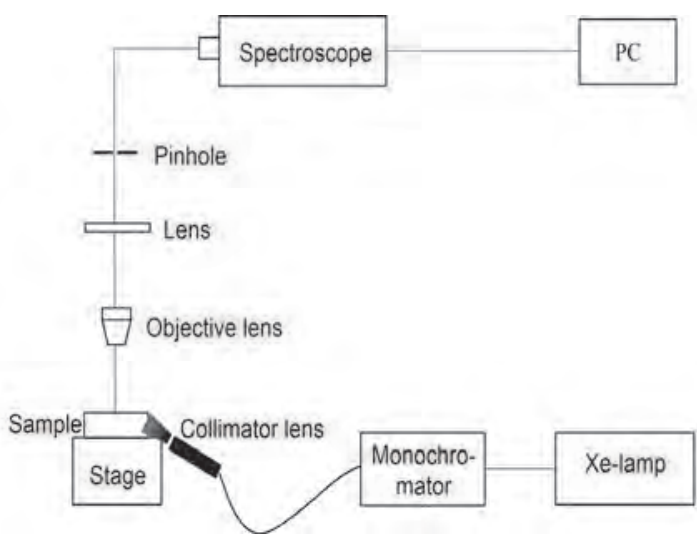

Fig. 1: The schematic illustration of the semiconfocal fluorescence microscope

Each part of the ScFM this microscope was checked of the wavelength range, wavelength accuracy, and spectral resolution to qualify the spectroscope, especially for measuring fluorescence emission spectra and of PAHs. The emission wavelength axis was calibrated with three main spectral lines of $365.015 \mathrm{~nm}, 404.656 \mathrm{~nm}$ and $435.833 \mathrm{~nm}$ of a mercury lamp (L937-01, Hamamatsu). The resolution of the spectroscope in UV region is evaluated to $1 \mathrm{~nm}$ of the full width at full width at half maximum (FWHM) for the $365.015 \mathrm{~nm}$ spectral line of mercury lamp.

All of the experiments described above were performed at room temperature.

\subsection{Chemicals}

Pyrene was selected as one typical example of PAHs. $1 \times 10^{-8} \mathrm{M}$ pyene aqueous solution was used as sample solution. Pyrene (purity, 99\%) was purchased from Janssen Chimica (Beerse, Belgium). Water was purified with water purification system (Milli-Q Academic A10, Millipore). $1 \times 10^{-6} \mathrm{M}$ pyrene aqueous solution was acquired directly from pyrene's saturated solution, and $1 \times 10^{-8} \mathrm{M}$ pyrene aqueous solution was acquired by diluting that of $1 \times 10^{-6} \mathrm{M}$ pyrene aqueous solution.

\section{Results and Discussion}

\subsection{Excitation light source}

A tunable monochromatic and ultraviolet light emitted from the light guide connected from the monochromator and the xenon lamp was assessed with the corrected spectroscope of ScFM. As shown in Fig. 2, the monochromatic light with a wavelength range of $260 \mathrm{~nm}$ to $400 \mathrm{~nm}$ are obtained: each emission spectrum of the light source is collected respectively under the condition of the CT-10 monochromator being set at $130.0 \mathrm{~nm}, 140.0$ $\mathrm{nm}, 150.0 \mathrm{~nm}, 160.0 \mathrm{~nm}, 170.0 \mathrm{~nm}, 180.0 \mathrm{~nm}, 190.0 \mathrm{~nm}$, or $200.0 \mathrm{~nm}$. This wavelength range fully covers absorption maxima of pyrene as well as a variety of PAHs. 


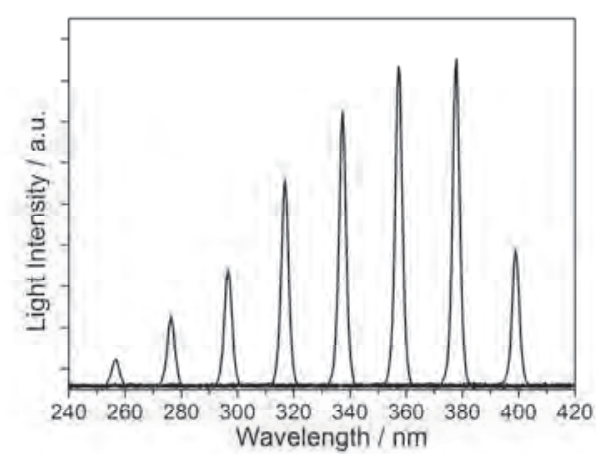

Fig. 2: The monochromatic light with a wavelength range of 260 to $400 \mathrm{~nm}$ obtained with the excitation light source.

The monochromaticity of ultraviolet light emitted from xenon lamp and monochromator was investigated. The spectrum of the monochromatic light at $357 \mathrm{~nm}$ is shown in Fig.3. Its monochromaticity was optimized by checking the dependence on the widths of the entrance and exit slits: i. e., for the monochromatic light at $357 \mathrm{~nm}$, the best resolution was obtained with the entrance and exit slit widths of $0.32 \mathrm{~mm}$ and $0.27 \mathrm{~mm}$, respectively. The FWHM of this ultraviolet monochromatic light is calculated to be $2.0 \mathrm{~nm}$, higher than $13 \mathrm{~nm}^{8)}$ and $5 \mathrm{~nm}^{9)}$ of other reported ones. This level of monochromaticity is enough to discuss dependence of the Stokes' shift on environment molecules feel. ${ }^{6}$ ) At $300 \mathrm{~nm}, 2 \mathrm{~nm}$ red shift corresponds to $220 \mathrm{~cm}^{-1}$ shift which is smaller than the typical values of Stokes' shift. The results show that the light source in ScFM developed can provide monochromatic ultraviolet light with $2.0 \mathrm{~nm}$ FWHM in the wavelength range between $260 \mathrm{~nm}$ and $400 \mathrm{~nm}$. In conclusion, the light source is successfully obtained for observing excitation spectra of PAHs adsorbed at the air/water interface with ScFM.

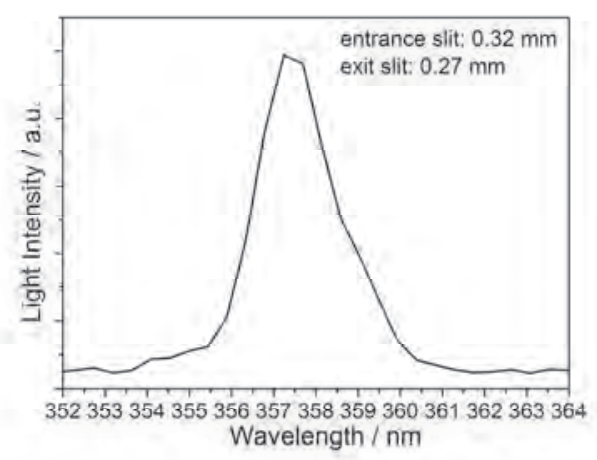

Fig. 3: The monochromatic light of $357 \mathrm{~nm}$ emitted from the xenon lamp and the monochromator.

\subsection{Fluorescence spectrum of pyrene molecules adsorbed at the air/water interface}

It is generally expected that ScFM has no good surfaceselectivity in observing fluorescence emission: fluorescence both from bulk solution and from surface is detected, and the contribution ratio $R_{f}$ of surface depends on surface activity of the target molecules and on thickness of the liquid solution, as shown in Eq. (1), ${ }^{5)}$ except for the fact that $d_{z}$ represents the solution thickness instead of the height of the probe volume.

$$
\begin{gathered}
R_{f}=\left(\eta_{s} \pi \omega^{2} N_{s}+\eta_{b} \pi \omega^{2} d_{z} C\right) /\left(2 \eta_{b} \pi \omega^{2} d_{z} C\right)= \\
\eta_{s} N_{s} / 2 \eta_{b} d_{z} C+1 / 2
\end{gathered}
$$

Where $\eta_{s}$ and $\eta_{b}$ are the efficiencies of fluorescence excitation-detection for fluorescence molecules at the air/water interface and in water bulk. $\omega$ is the radius of the cylinder-shaped probe volume of ScFM. $N_{s}$ is the surface density of targeted molecules. $C$ is the bulk concentration of target molecules.

Since $N_{S}=K N_{\max } C$, where $K$ is the adsorption equilibrium constant, and $N_{\max }$ is the maximum surface density of target molecules, Eq. (1) can be deformed into, ${ }^{5)}$

$$
R_{f}=\eta_{s} K N_{\max } / 2 \eta_{b} d_{z}+1 / 2
$$

As shown in Eq. (2), it is simply expected that surfaceselective observation is possible for target molecules with a high surface activity represented by $K$ or $N_{\max }$ and a small solution thickness $d_{z}$.

Figure 4 shows the fluorescence spectrum of pyrene molecules in the bulk solution measured with ScFM, excited by a band of mixed excitation light with a wavelength range between $310 \mathrm{~nm}$ and $360 \mathrm{~nm}$ from the excitation light source. This fluorescence spectrum was acquired under the experimental conditions of a large solution thickness of about $15 \mathrm{~mm}$ and the focus of objective lens into the water bulk. It is clear that this microscope can be used to observe fluorescence of PAHs.

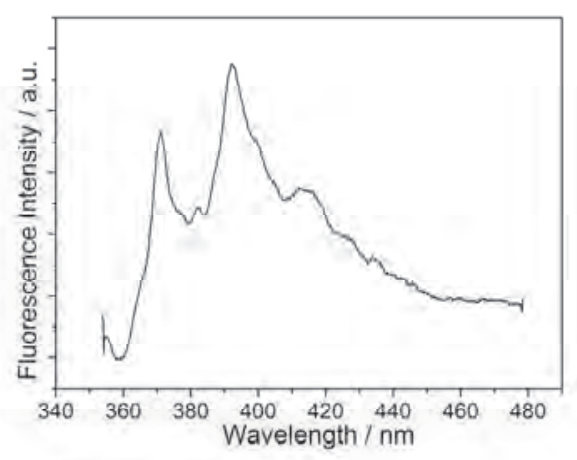

Fig. 4: The fluorescence spectrum of pyrene molecules in water bulk measured with the semi-confocal fluorescence microscope 
Figure 5 shows fluorescence spectra of pyrene molecules measured with ScFM under three different positions of the objective lens focusing. The position of 0 $\mu \mathrm{m}$ means that the focus of the objective lens is just at the water surface.

There was no significant position-dependence of fluorescence intensity as shown in Fig. 5. In order to distinguish fluorescence emissions of pyrene molecules adsorbed at the air/water interface from those in bulk, the ratio of fluorescence intensity at $391.1 \mathrm{~nm}$ with respect to that at $370.6 \mathrm{~nm}$ ( III/ I ratio) are calculated and summarized in Table 1. This III / I ratio is known to take 0.64 for pyrene in aqueous solution and 0.74 for pyrene adsorbed at the air/water interface. ${ }^{10)}$ The III/ I ratios in Tab. 1 are quite different from the reported ones, because they are uncorrected emission spectrum. However, the III / I ratios in Tab. 1 still do not affect us to conclude that these three fluorescence spectra of pyrene measured with ScFM are all dominated by pyrene molecules in the bulk solution. Although the thickness of pyrene solution is only $2 \mathrm{~mm}$, the intensity of fluorescence emissions of pyrene molecules in bulk was so high that it covers those adsorbed at the water surface. It is concluded that pyrene has a weak surface activity for observing fluorescence surface-selectively with ScFM developed.

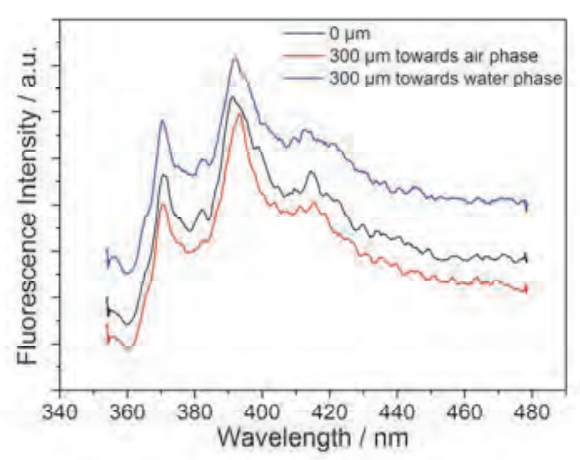

Fig. 5: Fluorescence spectra of pyrene molecules measured with the semi-confocal fluorescence microscope under different objective lens focusing positions.

Tab. 1: III/ I ratios of fluorescence spectra of pyrene molecules measured with semiconfocal fluorescence microscope

\begin{tabular}{cc}
\hline & III/ I ratio \\
\hline bulk & 1.024 \\
$0 \mu \mathrm{m}$ & 1.024 \\
$300 \mu \mathrm{m}$ (towards air phase) & 1.020 \\
$300 \mu \mathrm{m}$ (towards water phase ) & 1.017 \\
\hline
\end{tabular}

\section{Conclusion}

A semi-confocal fluorescence microscope is designed and constructed for observing fluorescence excitation spectrum of soluble molecules adsorbed at the air/water interface. A total internal reflection illumination of tunable monochromatic ultraviolet light from a xenon lamp is combined with a confocal fluorescence microscope. The range of wavelength and monochromaticity of the excitation light souse are examined. It can successfully provide monochromatic ultraviolet light with $2.0 \mathrm{~nm}$ FWHM in the wavelength range between $260 \mathrm{~nm}$ and $400 \mathrm{~nm}$. Theoretical feasibility is discussed to obtain fluorescence spectrum of molecules adsorbed at the air/water interface surface-selectively with the microscope. The fluorescence spectra of PAHs are observed with this microscope. It is found that pyrene has a weak surface activity for observing fluorescence surface-selectively with ScFM developed.

\section{Acknowledgements}

This work was financially supported by a Grant-in-Aid for challenging Exploratory Research [No. 23655067] from the Ministry of Education, Culture, Sports, Science and Technology. The authors greatly thank Dr. Kenji Furuya, Dr. Toshio Ishioka and Mrs. Miki Isoda for their kind help and discussion.

\section{References}

1) A. Harata, M. Sato, T. Ishioka, in Charged Particle and Photon Interactions with Matter: Recent Advances, Applications, and Interfaces, eds. by Y. Hatano, Y. Katsumura, A. Mozumder, CRC press, Boca Raton, p. 445 (2010).

2) G. L. Richmond, Chem. Rev., 102, 2693(2002).

3) Y. Li, Langmuir, 15, 3035 (1999).

4) Y. Li, T. Inoue, A. Harata, and T. Ogawa, Instrum. Sci. and Tech., 27, 159 (1999).

5) X. Zheng, A. Harata, and T. Ogawa, Specrtochim. Acta, Part A, 57, 315 (2001).

6) M. N. Slyadnev, T. Inoue, A. Harata, T. Ogawa, Colloids Surf., A, 164, 155 (2000).

7) J. Chen, F. S. Ehrenhauser, K. T. Valsaraj, and M. J. Wornat, J. Phys. Chem. A, 110, 9161 (2006).

8) K. Hirose, H. Sugahara, and H. Matsuno, J. Light \& Vis. Env., 26, 35 (2012).

9) R. J. Carman, D. M. Kane, and B. K. Ward, J. Phys. D: Appl. Phys. 43, 025205 (2010).

10)B. T. Mmereki, D. J. Donaldson, Phys. Chem. Chem. Phys., 4, 4186 (2002). 